\title{
Disrupted socio-emotional neural circuitry in adolescents with borderline personality
}

\section{traits}

Kristina Safar $^{\text {ab }}$, Julie Sato ${ }^{\text {abc }}$, Anthony C. Ruocco ${ }^{\text {d }}$, Marshall S. Korenblum ${ }^{\text {ef }}$, Helen $\mathrm{O}^{\prime} H_{a l p i n}{ }^{\text {ef }}$, Benjamin T. Dunkley ${ }^{\text {abgh }}$

${ }^{a}$ Diagnostic Imaging, Hospital for Sick Children, Toronto, Canada; ${ }^{b}$ Neurosciences and Mental Health Program, Research Institute, Hospital for Sick Children, Toronto, Canada; ${ }^{\mathrm{c}}$ Department of Psychology, University of Toronto, Toronto, Canada; ${ }^{\mathrm{d} D e p a r t m e n t ~ o f ~}$ Psychology, University of Toronto, Toronto, Canada; ${ }^{~}$ Department of Psychiatry, University of Toronto, Toronto, Canada; ${ }^{\mathrm{f} S i c k k i d s}$ Centre for Community Mental Health, Toronto, Canada; ' Department of Medical Imaging, Faculty of Medicine, University of Toronto, Toronto, Canada; ${ }^{\mathrm{h}}$ Institute of Medical Science, University of Toronto, Toronto, Canada. Corresponding author:

Kristina Safar, $\mathrm{PhD}$

Diagnostic Imaging, Hospital for Sick Children

555 University Avenue

Toronto, ON Canada M5G 1X8

Phone: 416-813-7654 x 309116

Email: kristina.safar@sickkids.ca

Running short title: Neural circuitry in adolescents with BPD traits

Keywords (6): borderline personality disorder; adolescence; magnetoencephalography; functional connectivity; phase synchrony; socio-emotional processing;

\section{Manuscript details:}

Abstract: 181 words; Main text: 3793 words; Figures: 3; Tables: 1; Supplementary Information: 0. 


\begin{abstract}
Background: Disruptions in fronto-limbic functional connectivity have been reported in adults with borderline personality disorder (BPD), although it is not yet known whether functional circuitry is similarly altered in adolescents with high levels of BPD traits.

Methods: Using magnetoencephalography (MEG), the current study investigated taskdependent functional connectivity of eight a priori regions of interest implicated in emotional and social processing during the implicit perception of emotional faces (angry and happy), in 8 female adolescents with BPD traits and 8 age-matched female controls.

Results: A network comprising reduced alpha-band phase synchrony during implicit angry face perception was observed in adolescents with BPD traits compared to controls. The network involved brain regions within a fronto-limbic circuit, including connections among bilateral amygdalae and bilateral anterior cingulate cortex (ACC), and additional regions implicated in emotion processing.
\end{abstract}

Conclusions: Results suggest that disrupted interregional oscillatory communication during the perception of threatening emotional expressions might contribute to the socio-emotional dysregulation and maladaptive behavioral responses commonly found in adolescents high in BPD traits. Furthermore, we speculate that this aberrant neural connectivity subserves the emergence of the disorder in adults.

\title{
Acknowledgments:
}

Financial disclosures: The authors have no biomedical financial or other conflicts of interest to declare. 
Neural circuitry in adolescents with BPD traits

\section{Introduction}

Emotion dysregulation is a core feature of borderline personality disorder (BPD) and is characterized by emotional vulnerability and difficulties regulating emotional responses $(1,2,3)$. Although the neurobiology of emotion dysregulation in BPD is not yet fully understood, a growing body of research suggests disruptions in the structure and function of fronto-limbic brain regions involved in socio-emotional processes (4).

Structural differences (e.g., abnormal gray matter volume) of several brain regions implicated in emotion perception and regulation have been observed in BPD (4). Aside from structural differences, adults with BPD demonstrate aberrant function of fronto-limbic brain regions in response to negative emotional stimuli (4-7). A meta-analysis of fMRI studies found greater activation of the bilateral posterior cingulate gyrus and right insula, as well as lower activation of the bilateral dorsolateral prefrontal cortex (PFC), right anterior cingulate cortex (ACC), right amygdala, and left superior temporal gyrus during negative emotion processing in BPD (5). Dysfunction of limbic areas, particularly the insula, was interpreted to reflect enhanced salience to and subjective experience of negative emotion, whereas disrupted activation of more anterior brain regions, including bilateral dorsolateral PFC and right ACC suggested difficulties in top down regulation of negative emotion, possibly because of disrupted connectivity between fronto-limbic regions (5). In a recent meta-analysis, Schulze et al. (6) also found greater activation of several limbic regions, including an area comprising the left amygdala and left hippocampus, along with the left insula in patients BPD when presented with negatively valenced stimuli. Patients with BPD also demonstrated attenuated activation in the bilateral dorsolateral PFC during negative affective processing in BPD. In particular, greater activation of the left amygdala was 
thought to reflect enhanced sensitivity to negative affect in BPD, whereas lower activation in frontal regions reflected difficulties in emotional control (6). Taken together, fMRI findings suggest disrupted function of frontal and subcortical limbic brain regions involved in the perception of negative stimuli and regulation of emotion in $\operatorname{BPD}(4,5,6)$.

Recent research investigating the interconnectedness of fronto-limbic circuitry has revealed differences in functional connectivity in BPD compared to controls during a resting-state and when presented with emotional stimuli (8-17). These studies also show disrupted fronto-limbic networks, which play a fundamental role in processing emotional information. For instance, lower coupling of glucose metabolic activity between the amygdala and orbitofrontal cortex at rest has been observed in BPD (8). During processing of emotional stimuli, Dudas et al. (9) reported greater functional connectivity when viewing emotion-inducing images depicting disgust in adults with BPD between the left amygdala and left dorsolateral PFC, and left amygdala and ventral striatum. Cullen et al. (10) examined functional connectivity of the amygdala to neutral, overt fearful, and masked fearful facial expressions, finding lower connectivity between bilateral amygdalae and midcingulate cortex to neutral faces in BPD. In contrast, BPD showed higher bilateral amygdalae and ACC coupling to overt fearful faces, and higher right amygdala and bilateral thalamus and caudate coupling to masked fear faces. Additionally, higher amygdala connectivity was positively correlated with clinical measures of BPD. Lower functional connectivity in BPD during neutral faces was interpreted to reflect disrupted amygdala circuitry during non-emotional contexts, which may bring about compensatory higher amygdala connectivity during fearful face processing (10). 
Whereas most neuroimaging research on BPD has been carried out on adults, BPD has more recently been recognized as a valid and reliable diagnosis in adolescence (18). Accordingly, the neurobiology underlying emotion dysregulation in adolescents with BPD has been studied less frequently. Many studies have revealed lower grey matter volumes in right amygdala, bilateral hippocampi and left ACC in adolescents with BPD $(19,20)$, yet structural atypicalities are not consistently replicated across studies $(21,22)$. In addition, lower grey matter volume in bilateral dorsolateral PFC and left OFC have been observed in adolescents with BPD compared to controls (21). Only one pilot neuroimaging study has examined functional activation of brain regions in adolescents with BPD (23). Using fMRI, LeBoeuf et al. (23) found increased activation in the right amygdala, right hippocampus and parahippocampus, bilateral superior frontal gyri, right precentral gyrus and cerebellum in adolescents with BPD during the presentation of negative images, whereas higher activation in the left precentral gyrus and right orbitofrontal gyrus was found in controls. Thus, aberrant activation of fronto-limbic brain regions importantly involved in affect perception and regulation is similarly present in adolescents and adults with BPD (23).

It is not yet known whether functional connectivity is disrupted in adolescents with BPD - uncovering this would advance the neurobiological understanding of the disorder and identify biomarkers that confer a risk for developing BPD. Electrophysiological imaging, such as magnetoencephalography (MEG), offers both an excellent temporal and good spatial resolution (24), and directly measures multidimensional neurophysiology that is not captured in haemodynamics. The current study investigated task-dependent functional connectivity of eight regions of interest (ROIs) underlying emotional and social processing during the implicit perception of expressive faces in female adolescents with BPD traits. We 
focused on females because this group is more likely to be diagnosed with BPD compared to males in adolescent clinical samples (25), reducing sample heterogeneity and eliminating sex as a confounding factor. Selected ROIs were the bilateral superior temporal gyri, amygdalae, insulae and ACC.

We hypothesized that functional connectivity among the eight ROIs, as well as connections between these regions and prefrontal areas, would be lower in female adolescents high in BPD traits compared to age- and sex-matched controls during processing of negative affect. In particular, we focused on angry faces because difficulties with the regulation of negative affect, and especially anger, are central to the diagnosis of BPD (26). 
Neural circuitry in adolescents with BPD traits

\section{Methods and Materials}

Participants

Eight female adolescents with BPD traits $(M$ age $=15.96$ years, $S D=1.01$, range: $14.38-17$ years $)$ and eight female controls $(M$ age $=15.11$ years, $S D=1.43$, range: $13.68-$ 18.21 years), who were matched on age, $t(14)=1.37, p=.192$, participated. Participants. BPD traits were assessed using the Structured Interview for DSM-IV Personality (SIDPIV)—BPD Module (27). The SIDP-IV is a semi-structured interview that assesses all DSMIV criteria for BPD on a scale from 0 (not present or limited to rare isolated examples) to 3 (strongly present-criterion is associated with subjective distress or impairment in social or occupational functioning or intimate relationships) with strong reliability and validity for assessing BPD (28). The mean number of criteria met for the BPD group was 6.75 (SD = 1.83). The percentage of endorsement for each of the SIDP-IV BPD criteria were as follows: $100 \%$ affective instability and suicidal/self-mutilating behaviour; $87.5 \%$ unstable interpersonal relationships, difficulty controlling anger, and feelings of emptiness; $75 \%$ efforts to avoid abandonment; $62.5 \%$ impulsivity; and $37.5 \%$ identity disturbance and dissociative symptoms. The symptoms were required to be present for at least two years prior as evidence of sufficient temporal stability. At the time of testing, $75 \%$ of adolescents with BPD traits were taking medications: antipsychotic $(33.3 \%)$ or antidepressant $(66.6 \%)$ medications. Treating clinician diagnoses for adolescents with BPD traits included: $12.5 \%$ oppositional defiant disorder, $12.5 \%$ posttraumatic stress disorder, $37.5 \%$ major depressive disorder and posttraumatic stress disorder, $12.5 \%$ posttraumatic stress disorder and dysthymic disorder, $12.5 \%$ conduct disorder, and $12.5 \%$ major depressive disorder and generalized anxiety disorder. Participants were ineligible for the study if they met DSM-IV 
criteria for autism spectrum disorder or neurological disorders, mental retardation, or had metal implants that could not be removed. The study was approved by the Hospital for Sick Children Research Ethics Board and written informed assent/consent was obtained from all participants and their parents.

Emotional faces task and MEG data acquisition

The emotional faces task is described in Leung and colleagues (29). Angry and happy faces ( 25 faces, 13 males) with validity ratings of 0.8 proportion correct or higher were chosen from the MacBrain Face Stimulus Set (30) available at www.macbrain.org. MEG was recorded while participants saw a happy or angry face and the corresponding scrambled pattern (target stimulus) simultaneously on either side of a central fixation cross. To generate corresponding scrambled patterns matched for visual properties (i.e., luminance and color), a mosaic filter was applied to the faces to using Photoshop (Adobe Systems Inc., San Jose, CA), and the faces were parcelled into 64 square tiles, which were then shuffled and Gaussian blurred (10.0 pixels). Adolescents indicated the side of the target by pressing a button (left or right) as rapidly as possible.

Each trial consisted of $80 \mathrm{~ms}$ stimulus presentation followed by an inter-stimulus interval jittered between 1300 and 1500 ms. Participants were presented with 50 trials per face type, presented twice each in the left and right hemifields, in randomized order (200 happy and angry trials in total). Stimuli were presented using Presentation ${ }^{\circledR}$ (Neurobehavioral Systems, Inc., Berkeley, CA, www.neurobs.com) at a viewing distance of approximately $75 \mathrm{~cm}$ and subtended approximately 6.9 degrees of visual angle (Figure 1). 


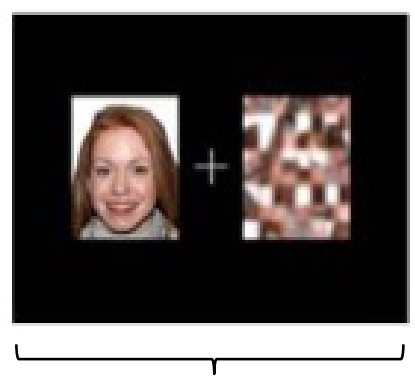

$80 \mathrm{~ms}$

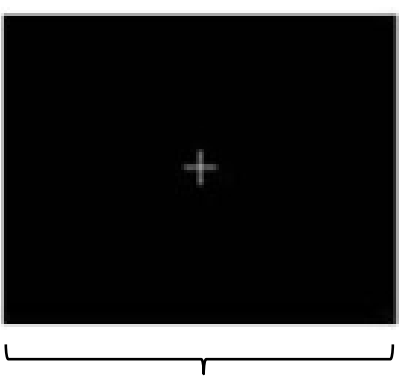

$1300-1500 \mathrm{~ms}$

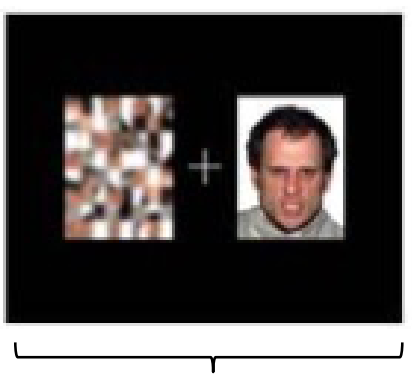

$80 \mathrm{~ms}$

$\longleftarrow$ Response

Figure 1. Emotional Faces Task. Adolescents saw a happy or angry face and corresponding scrambled pattern (target) simultaneously on either side of a central fixation cross, and rapidly indicated the left or right position of the target.

MEG data was acquired in a magnetically shielded room while participants were supine, on a 151 channel CTF system at a $600 \mathrm{~Hz}$ sampling rate. Head location was continuously recorded by fiducial coils placed at the left and right pre-auricular points and the nasion. A T1-weighted MRI scan (3D SAG MPRAGE, GRAPPA =2, TR/TE/FA $=2300 \mathrm{~ms} / 2.96 \mathrm{~ms} / 90^{\circ}, \mathrm{FOV}=28.8 \times 19.2 \mathrm{~cm}, 256 \times 256$ matrix, 192 slices, slice thickness $=1.0 \mathrm{~mm}$ isotropic voxels) was obtained for each participant on a 3T MRI scanner (MAGNETOM Tim Trio, Siemens AG, Erlangen, Germany) with a 12-channel head coil. The placement of the fiducial coils was later used as reference for MEG data coregistration.

\section{MEG data pre-processing and source reconstruction}

MEG data was epoched into -500 to $1500 \mathrm{~ms}$ segments relative to stimulus onset, and divided into face type (angry or happy). Epoched data trials with head motion exceeding $5 \mathrm{~mm}$ within and between each trial were excluded from analysis. All trials were visually scanned for artefacts (i.e., eye movement, blinks) and excluded if necessary. The FieldTrip MATLAB software toolbox (31) was used for MEG data processing. A single 
shell head model was computed from each participant's individual MRI and was normalized onto a standardized ICBM 152 template (32). We used the first 90 sources (seeds), which includes cortical and subcortical brain regions defined in the Automated Anatomical Labelling (AAL) atlas (33). These sources were unwarped from the standardized brain template space onto corresponding locations for each participant's headspace (33). The broadband $(1-150 \mathrm{~Hz})$ time-series for these source locations was reconstructed for each trial using a linearly constrained minimum variance (LCMV) beamformer (34). Beamforming is an adaptive spatial filtering technique that is used to estimate source activity at a region of interest while attenuating activity from other sources i.e. regions not of interest, or environmental and physiological artifacts (e.g., ocular and muscle; 35).

\section{Phase synchrony}

The reconstructed time-series data for each source location were filtered into theta (4-7 Hz), alpha $(8-14 \mathrm{~Hz})$ and beta $(15-30 \mathrm{~Hz})$ canonical frequency-bands. The Hilbert Transform was calculated to attain instantaneous value of phase for each source and frequency band.

Phase synchrony of ongoing neural oscillations between brain regions was indexed using the cross-trial phase-lag index (PLI) based on Stam, Nolte and Daffertshofer (36). The PLI estimates phase synchrony between the time-series of two sources by calculating the consistency of their phase relationship, while attenuating spurious connectivity estimates driven by zero or near-zero phase lag as a result of volume conduction and/or signal leakage (36). PLI was calculated between the eight selected sources (bilateral amygdalae, STG, insulae and ACC) and 90 sources covering a distributed grid across the brain, yielding a 90 x 8 connectivity matrix for each time/sample point, across trials for each of the face types in 
Neural circuitry in adolescents with BPD traits

each of the frequency-bands. At each time point, PLI values were z-scored relative to a baseline period of -500 to $0 \mathrm{~ms}$ prior to stimulus onset.

\section{The Network Based Statistic}

An active time window of phase synchrony between $0-400 \mathrm{~ms}$ following stimulus onset for each participant was selected for statistical analysis using the Network Based Statistic (NBS; 37, 38). We selected this active time window based on previous research indicating differences in phase synchronization 0 - $400 \mathrm{~ms}$ after stimulus presentation using the same task (29). NBS is a non-parametric technique to identify significant differences in network connectivity while controlling for family wise error rate (FWE; 37, 38). NBS first applies a test statistic threshold (a $t$-test in this case) for every edge in the connectivity matrix. Components (i.e., contiguous clusters of supra-threshold connections between any two or more brain sources exceeding the statistical threshold) are then identified and subjected to random permutation testing to evaluate statistical significance. For each permutation (5,000 permutations in the current study), group membership is randomly shuffled and the largest component observed is recorded to create a null distribution. The null distribution is then compared to the size of the originally identified, empirically derived, components to assess statistical significance and a $p$-value is assigned accordingly $(37,38)$. As recommended by Zalesky and colleagues $(37,38)$, for the alpha frequencyband we adapted the t-test statistical threshold for within-group comparisons analysis to $\mathrm{t}=$ 2.1 and for between-group comparisons analysis to $\mathrm{t}=2.3$.

\section{Correlations with symptom measures}

Following the NBS analysis, correlations were computed for connectivity strength of specific ROIs identified by NBS as part of a network that significantly differed between- 
groups and symptom measures in adolescents with BPD traits. The Brain Connectivity Toolbox was used to compute connectivity strength, which is the sum of a specific region's connections to all other brain regions and a measure of the importance of that region in a network (39). Strength was computed for each time point across the active time window (0 - $400 \mathrm{~ms}$ ) for these ROIs. Benjamini-Hochberg adjusted p-values were used to correct for multiple comparisons $\left(p_{\mathrm{BH}}<.05 ; 40\right)$. 
Neural circuitry in adolescents with BPD traits

\section{Results}

\section{Behavioral performance}

The Wechsler Abbreviated Scale of Intelligence (WASI; 41) was used to assess fullscale IQ. Full-scale IQ scores did not significantly differ between-groups, $t(14)=.412, p=$ .687. To measure dimensions of emotion dysregulation, adolescents with BPD traits completed the Difficulties in Emotion Regulation Scale (DERS; 42). The mean total score for adolescents with BPD traits was $2.72(S D=.68$, range $=1.72-3.61)$.

No significant difference between adolescents with BPD traits and controls in reaction time $(\mathrm{ms})$ to happy faces $t(14)=.182, p=.858$, or angry faces $t(14)=.265, p=$ .795) was revealed. A Mann-Whitney test was calculated due to non-normality of the distribution for accuracy (percent correct) as indicated by a Shapiro-Wilk test for happy faces $(w(16)=.864, p=.022)$ and angry faces $(w(16)=.795, p=.002)$. No significant difference between adolescents with BPD traits and controls in accuracy for happy faces, $U$ $=36.5, z=.474, p=.645$, or angry faces $U=38, z=.633, p=.574$.

\section{Phase synchrony}

Increased within-group alpha-band phase synchrony

Significant increases in phase synchrony in the alpha frequency-band were observed within both BPD and control groups, 0 - 400 ms following stimulus onset relative to baseline during processing of happy and angry faces. For both groups, networks of increased phase synchrony encompassed interregional connections involving a priori ROIs and occipital, parietal, temporal and frontal brain regions, as well as subcortical limbic areas key for emotional face processing (Figure 2; Table 1). During presentation of angry faces, adolescents with BPD traits showed a network of increased task-dependent phase synchrony 
involving interregional connections involving six ROIs (bilateral superior temporal gyri and bilateral ACC, left amygdala and left insula; 26 edges and 24 nodes, $p=.017)$. Similarly, a network of increased task-dependent phase synchrony was revealed in adolescents with BPD traits to happy faces, involving widespread connections between six ROIs (right superior temporal gyrus, bilateral ACC, bilateral amygdalae, and right insula; 23 edges and 23 nodes, $p=.01)$. For controls, increased phase synchrony during the presentation of angry faces relative to baseline was found. This network included six ROIs (bilateral ACC, bilateral amygdalae, bilateral insulae; 29 edges and 25 nodes, $p=.012$ ). Likewise, to happy faces a network of extensive increased task-dependent phase synchrony was revealed encompassing five ROIs (bilateral superior temporal gyri, left ACC, bilateral insulae; 32 edges, and 28 nodes, $p=.004)$.

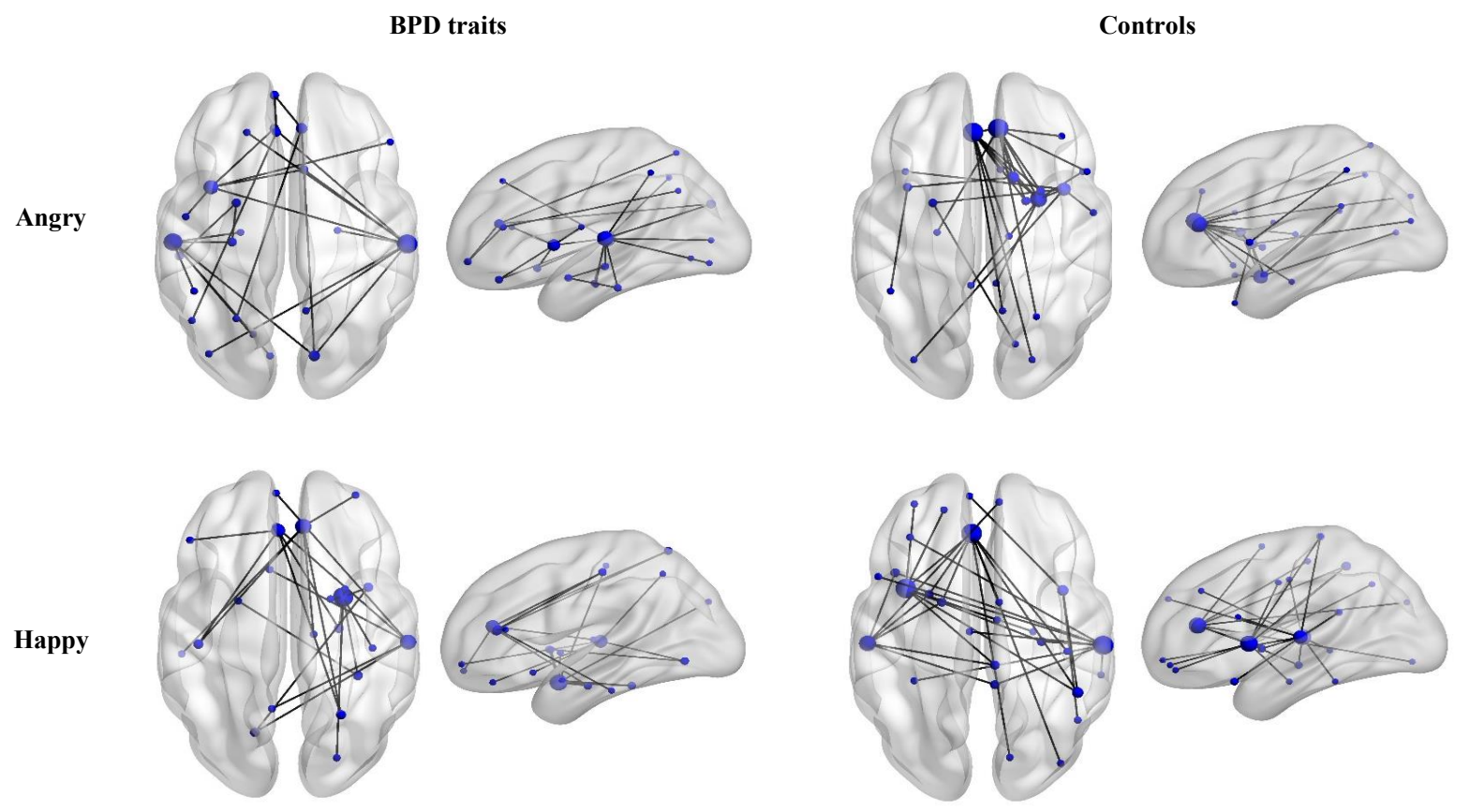

Figure 2. Increased within-group alpha-band phase synchrony 0 - $400 \mathrm{~ms}$ following angry and happy face onset relative to baseline in adolescents with BPD traits and controls. In adolescents with BPD traits and controls, networks comprised widespread connections involving a priori ROIs and occipital, parietal, temporal and frontal brain regions, as well as subcortical limbic areas important for processing emotional faces. Node size is scaled by the number of connected nodes (degree) in each of the networks. 


\section{Reduced alpha-band phase synchrony in BPD}

Reduced phase synchrony in the alpha frequency-band was revealed in adolescents with BPD traits compared to controls during angry face processing, $0-400 \mathrm{~ms}$ following stimulus onset. This network of reduced phase synchrony in adolescents with BPD traits included interregional connections among four of the eight ROIs (bilateral amygdalae and bilateral ACC) and other core emotional face processing regions. Specifically, this network comprised 19 edges and 14 nodes $\left(p_{\text {corr }}=.031\right)$. Of particular interest, reduced phase synchronization was found between the right amygdala and frontal regions, including the bilateral ACC, the right dorsolateral superior frontal gyrus, and the right superior frontal gyrus (medial part). In addition, reduced connectivity between the right pallidum and the bilateral ACC was observed, along with decreased connectivity between frontal regions, involving the left ACC and the right middle frontal gyrus (orbital part) and the right superior frontal gyrus (orbital part). Each of the regions and connections in this network are displayed in Figure 3, and listed in Table 2. No significant between- group differences were found for happy faces in the alpha frequency-band, or for either face type in the theta and beta frequency-bands. 


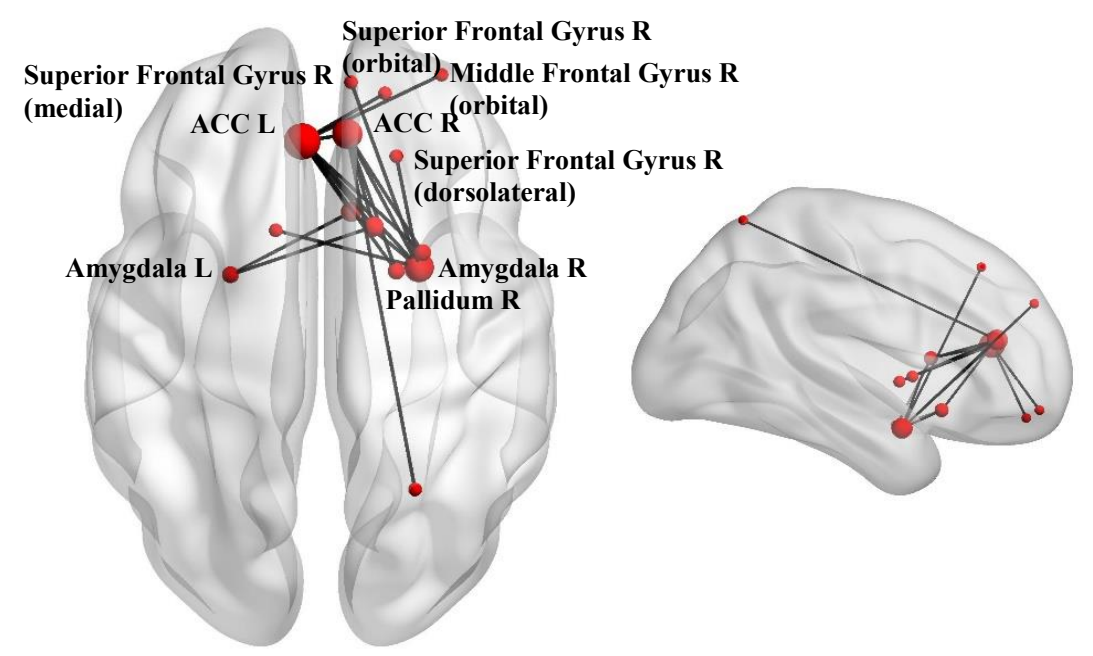

Figure 3. Reduced alpha-band phase synchrony during angry face processing $0-400 \mathrm{~ms}$ in adolescents with BPD traits compared to controls. This network comprised 14 nodes, and 19 edges, $p_{\text {corr }}=.031$. Node size is scaled by the number of connected nodes (degree) of this network.

\begin{tabular}{ll}
\hline \multicolumn{1}{l}{ Region } & $\begin{array}{l}\text { Number of } \\
\text { Connections (degree) }\end{array}$ \\
\hline Frontal superior gyrus R (dorsolateral) & 1 \\
Frontal superior gyrus R (orbital) & 1 \\
$\begin{array}{l}\text { Middle frontal gyrus R } \\
\text { (orbital) }\end{array}$ & 1 \\
Olfactory gyrus R & 3 \\
Superior frontal gyrus R (medial) & 1 \\
Anterior cingulate cortex L & 8 \\
Anterior cingulate cortex R & 6 \\
Amygdala L & 2 \\
Amygdala R & 6 \\
Superior parietal gyrus R & 1 \\
Caudate L & 1 \\
Caudate R & 3 \\
Putamen R & 2 \\
Pallidum R & 2 \\
\hline
\end{tabular}

Table 1. Brain regions involved in hypoconnected alpha-band circuitry in adolescents with BPD traits compared to controls during the first $400 \mathrm{~ms}$ of angry face processing and the number of connections. 
Strength correlations with symptom measures

Brain-behaviour correlations were also examined to determine whether alpha-band connectivity strength of the bilateral amygdalae and the bilateral ACC (brain regions revealed as significantly hypo-connected in adolescents in BPD vs. controls in the NBS analysis) during implicit angry face processing is correlated with BPD symptomology. Specifically, correlations between alpha-band connectivity strength during angry face processing and outcomes on the BPD Module from SIDP-IV including: ratings on difficulty controlling anger and affective instability items, total number of symptoms endorsed, and sum of ratings across all items. We also examined correlations between alpha-band connectivity strength and total DERS scores. Alpha connectivity strength during angry face processing in each of these regions did not significantly correlate with the SIDP-IV, or DERS outcomes in adolescents with BPD (all $\left.p_{\mathrm{BH}}>.05\right)$. 
Neural circuitry in adolescents with BPD traits

\section{Discussion}

Using MEG, the present study investigated functional connectivity of eight ROIs implicated in emotional face processing in female adolescents with BPD traits. Our findings show reduced phase synchrony in the alpha frequency-band following the presentation of implicit angry faces in adolescents with BPD traits compared to controls. This network involved several connections between fronto-limbic regions important for emotional face processing, including the right amygdala and the bilateral ACC, right dorsolateral superior frontal gyrus, and the medial part of the right superior frontal gyrus. Furthermore, reduced connectivity among the bilateral ACC and right pallidum, as well as connections between the left ACC and right middle frontal gyrus (orbital part) and right superior frontal gyrus (orbital part). Results are consistent with previous work reporting structural deficits and dysfunction of fronto-limbic brain regions during the presentation of negative emotional stimuli in adolescents and adults with $\operatorname{BPD}(4-7,21,22,23)$ and extend previous literature outlining aberrations in functional connectivity in adults with $\operatorname{BPD}(14,15)$.

In particular, the current findings indicate disrupted circuitry among bilateral amygdalae and frontal brain regions during the perception of threatening stimuli in adolescents with BPD traits. Reduced fronto-amygdala circuitry has been reported previously in adults with $\operatorname{BPD}(8,10,12,17,43)$, such as positron emission tomography (PET) results that indicate reduced metabolic correlations between the ventral amygdala and right orbitofrontal cortex in adults with BPD, that is thought to reflect an impaired ability to down regulate the amygdala in response to negative emotion in BPD (8). Furthermore, patterns of temporally early delayed gamma phase synchrony over posterior regions, and temporally late reduced gamma phase synchrony over the right hemisphere following onset 
of salient tones, have been observed in adults with BPD, suggesting attenuated functional integration in areas critical for the employment of attentional processing of salient stimuli and subsequent evaluation/discrimination of task-relevant information, respectively (43). Moreover, following an effortful emotion regulation task, adults with BPD demonstrated a lack of increase in resting-state functional connectivity between the amygdala and several other brain regions (i.e., left superior temporal gyrus and prefrontal areas) critically implicated in affective and cognitive control compared to non-psychiatric controls (11). Our results are consistent with this body of research. Given the importance of phase synchrony for interactions between populations of neurons $(44,45)$, our results suggest disrupted communication of subcortical limbic and frontal regions, including the amygdala, involved in the early detection and evaluation of negative emotion (46); the ACC, implicated in attention and control of emotional response (47); and areas of the PFC, critical for conceptual knowledge, regulation and response inhibition $(46,48)$. It is possible that this disrupted neural circuitry may underpin the emergence of the disorder in adults.

Reduced neurophysiological connectivity is consistent with structural studies in adolescents with BPD that have reported abnormal grey matter volume of brain regions part of this network compared to controls (i.e., right amygdala, left ACC, bilateral dorsolateral PFC and left OFC; see Winsper et al. (49) for a systematic review and meta-analysis). These structural differences have been associated with measures of BPD symptoms, such as suicidal behavior and impulsivity $(20,50)$. Given that functional networks are supported by structural architecture (51), it is possible that disconnection of fronto-limbic regions may be associated with key features of emotion dysregulation in adolescents with BPD traits. Although we did not find significant correlations of connectivity strength with BPD 
symptoms or dimensions of emotion dysregulation in the BPD group, future research might explore these associations in larger samples.

Our findings of reduced functional connectivity in adolescents with BPD were specific to the alpha frequency-band. Alpha-band synchrony plays an integrative role in a myriad of perceptual and cognitive processes $(52,53)$. For instance, alpha-band phase synchrony is pertinent to the perception and recognition of meaningful objects (52-56). It is possible that alpha synchrony may be importantly implicated in the perception and recognition of angry faces, which is disrupted in adolescents with BPD traits.

In the present study, the use of MEG identified disrupted fronto-limbic circuitry during the implicit perception of threatening emotional expressions in adolescents with BPD traits. Our results suggest disrupted interregional spectral communication among subcortical limbic brain regions important for early perceptual processing, as well as frontal regions critical for higher-level processing and regulation of affective information, which may explain emotional dysregulation evident in this vulnerable population. 


\section{References}

1. Linehan, M. (1993). Cognitive-behavioral treatment of borderline personality disorder. Guilford press.

2. Wagner, A.W., \& Linehan, M.M. (1999). Facial expression recognition ability among women with borderline personality disorder: implications for emotion regulation? Journal of Personality Disorders, 13, 329-344.

3. Zanarini, M.C., \& Frankenburg, F.R. (2007). The essential nature of borderline psychopathology. Journal of Personality Disorders, 21, 518-535.

4. Ruocco, A. C., \& Carcone, D. (2016). A neurobiological model of borderline personality disorder: systematic and integrative review. Harvard Review of Psychiatry, 24, 311-329.

5. Ruocco, A.C., Amirthavasagam, S., Choi-Kain, L.W., \& McMain, S.F. (2013). Neural correlates of negative emotionality in borderline personality disorder: An activation-likelihoodestimation meta-analysis. Biological Psychiatry, 73, 153-160.

6. Schulze, L., Schmahl, C., \& Niedtfeld, I. (2016). Neural correlates of disturbed emotion processing in borderline personality disorder: a multimodal meta-analysis. Biological Psychiatry, 79, 97-106.

7. van Zutphen, L., Siep, N., Jacob, G. A., Goebel, R., \& Arntz, A. (2015). Emotional sensitivity, emotion regulation and impulsivity in borderline personality disorder: A critical review of fMRI studies. Neuroscience \& Biobehavioral Reviews, 51, 64-76.

8. New, A. S., Hazlett, E. A., Buchsbaum, M. S., Goodman, M., Mitelman, S. A., Newmark, R., Trisdorfer, R., Haznedar, M., Koenigsberg, H.W., Flory, J. \& Siever, L.J. (2007). Amygdalaprefrontal disconnection in borderline personality disorder. Neuropsychopharmacology, 32, 1629. 
9. Dudas, R.B., Mole, T.B., Morris, L.S., Denman, C., Hill, E., Szalma, B., Evans, D., Dunn, B., Fletcger, P. \& Voon, V. (2017). Amygdala and dlPFC abnormalities, with aberrant connectivity and habituation in response to emotional stimuli in females with BPD. Journal of Affective Disorders, 208, 460-466.

10. Cullen, K.R., Vizueta, N., Thomas, K.M., Han, G.J., Lim, K.O., Camchong, J., Mueller, B.A., Bell, C.H., Heller, M.D. \& Schulz, S. C. (2011). Amygdala functional connectivity in young women with borderline personality disorder. Brain Connectivity, 1, 61-71.

11. Baczkowski, B.M., van Zutphen, L., Siep, N., Jacob, G.A., Domes, G., Maier, S., Sprenger, A., Willenborg, B., Tüscher, O., Arntz, A. \& van de Ven, V. (2017). Deficient amygdala-prefrontal intrinsic connectivity after effortful emotion regulation in borderline personality disorder. European Archives of Psychiatry and Clinical Neuroscience, 267, 551-565.

12. Doll, A., Sorg, C., Manoliu, A., Meng, C., Wöller, A., Förstl, H., Zimmer, C., Wohlschläger, A.M. \& Riedl, V. (2013). Shifted intrinsic connectivity of central executive and salience network in borderline personality disorder. Frontiers in Human Neuroscience, 7, 727.

13. Kamphausen, S., Schröder, P., Maier, S., Bader, K., Feige, B., Kaller, C. P., Glauche, V., Ohlendorf, S., Tebartz Van Elst, L., Klöppel, S., Jacob, G.A., Silberseig, D., Lieb, K. \& Tüscher, O. (2012). Medial prefrontal dysfunction and prolonged amygdala response during instructed fear processing in borderline personality disorder. The World Journal of Biological Psychiatry, 14, 307-318.

14. Krause-Utz, A., Elzinga, B.M., Oei, N.Y., Paret, C., Niedtfeld, I., Spinhoven, P., Bohus, M. \& Schmahl, C. (2014a). Amygdala and dorsal anterior cingulate connectivity during an emotional working memory task in borderline personality disorder patients with interpersonal trauma history. Frontiers in Human Neuroscience, 8, 848. 
15. Krause-Utz, A., Winter, D., Niedtfeld, I., \& Schmahl, C. (2014b). The latest neuroimaging findings in borderline personality disorder. Current Psychiatry Reports, 16, 1-13.

16. Soloff, P., Nutche, J., Goradia, D., \& Diwadkar, V. (2008). Structural brain abnormalities in borderline personality disorder: a voxel-based morphometry study. Psychiatry Research: Neuroimaging, 164, 223-236.

17. Wolf, R.C., Sambataro, F., Vasic, N., Schmid, M., Thomann, P.A., Bienentreu, S.D., \& Wolf, N.D. (2011). Aberrant connectivity of resting-state networks in borderline personality disorder. Journal of Psychiatry \& Neuroscience, 36, 402-411.

18. Kaess, M., Brunner, R., \& Chanen, A. (2014). Borderline personality disorder in adolescence. Pediatrics, 134, 782-793.

19. Richter, J., Brunner, R., Parzer, P., Resch, F., Stieltjes, B., \& Henze, R. (2014). Reduced cortical and subcortical volumes in female adolescents with borderline personality disorder. Psychiatry Research: Neuroimaging, 221, 179-186.

20. Whittle, S., Chanen, A.M., Fornito, A., McGorry, P.D., Pantelis, C., \& Yücel, M. (2009). Anterior cingulate volume in adolescents with first-presentation borderline personality disorder. Psychiatry Research: Neuroimaging, 172, 155-160.

21. Brunner, R., Henze, R., Parzer, P., Kramer, J., Feigl, N., Lutz, K., Essig, M., Resch, F. \& Stieltjes, B. (2010). Reduced prefrontal and orbitofrontal gray matter in female adolescents with borderline personality disorder: Is it disorder specific? Neuroimage, 49, 114-120.

22. Chanen, A. M., Velakoulis, D., Carison, K., Gaunson, K., Wood, S. J., Yuen, H. P., Yücel, M., Jackson, H.J., McGorry, P.D. \& Pantelis, C. (2008). Orbitofrontal, amygdala and hippocampal volumes in teenagers with first-presentation borderline personality disorder. Psychiatry Research: Neuroimaging, 163, 116-125. 
23. LeBoeuf, A., Guile, J. M., Labelle, R., \& Luck, D. (2017). Functional Neuroimaging Pilot Study of Borderline Personality Disorder in Adolescents. Sante Mentale au Quebec, 41, 141162.

24. Hämäläinen, M., Hari, R., Ilmoniemi, R.J., Knuutila, J., \& Lounasmaa, O.V. (1993). Magnetoencephalography - theory, instrumentation, and applications to noninvasive studies of the working human brain. Reviews of Modern Physics, 65, 413.

25. Miller, A.L., Muehlenkamp, J.J., \& Jacobson, C.M. (2008). Fact or fiction: Diagnosing borderline personality disorder in adolescents. Clinical Psychology Review, 28, 969-981.

26. American Psychiatric Association. (2013). Diagnostic and statistical manual of mental disorders (DSM-5®). American Psychiatric Publishing.

27. Pfohl, B., Blum, N., \& Zimmerman, M. (1997). Structured interview for DSM-IV personality: Sidp-IV. American Psychiatric Pub.

28. Carcone, D., Tokarz, V.L., \& Ruocco, A.C. (2015). A systematic review on the reliability and validity of semistructured diagnostic interviews for borderline personality disorder. Canadian Psychology/Psychologie Canadienne, 56, 208-226.

29. Leung, R.C., Annette, X.Y., Wong, S.M., Taylor, M.J., \& Doesburg, S.M. (2014). Reduced beta connectivity during emotional face processing in adolescents with autism. Molecular Autism, 5, 51.

30. Tottenham, N., Tanaka, J.W., Leon, A. C., McCarry, T., Nurse, M., Hare, T. A., Marcus, D.J., Westerlund, A., Casey, B.J. \& Nelson, C. (2009). The NimStim set of facial expressions: judgments from untrained research participants. Psychiatry Research, 168, 242-249.

31. Oostenveld, R., Fries, P., Maris, E., \& Schoffelen, J.M. (2011). FieldTrip: Open source software for advanced analysis of MEG, EEG, and invasive electrophysiological 
data. Computational Intelligence and Neuroscience, 2011, 1.

32. Fonov, V., Evans, A.C., Botteron, K., Almli, C.R., McKinstry, R.C., Collins, D.L., \& Brain Development Cooperative Group. (2011). Unbiased average age-appropriate atlases for pediatric studies. Neuroimage, 54, 313-327.

33. Tzourio-Mazoyer, N., Landeau, B., Papathanassiou, D., Crivello, F., Etard, O., Delcroix, N., Mazoyer, B., \& Joliot, M. (2002). Automated anatomical labeling of activations in SPM using a macroscopic anatomical parcellation of the MNI MRI single-subject brain. Neuroimage, 15, 273-289.

34. Van Veen, B., van Drongelen, W., Yuchtman, M., Suzuki, A. (1997). Localization of brain electrical activity via linearly constrained minimum variance spatial filtering. IEEE Transactions on Biomedical Engineering. 44, 867-80.

35. Doesburg, S. M., Vidal, J., \& Taylor, M. J. (2013). Reduced theta connectivity during setshifting in children with autism. Frontiers in Human Neuroscience, 7, 192-201.

36. Stam, C. J., Nolte, G., \& Daffertshofer, A. (2007). Phase lag index: assessment of functional connectivity from multichannel EEG and MEG with diminished bias from common sources. Human Brain Mapping, 28, 1178-1193.

37. Zalesky, A., Cocchi, L., Fornito, A., Murray, M. M., \& Bullmore, E. D. (2012). Connectivity differences in brain networks. Neuroimage, 60, 1055-1062.

38. Zalesky, A., Fornito, A., \& Bullmore, E. T. (2010). Network-based statistic: identifying differences in brain networks. Neuroimage, 53, 1197-1207.

39. Rubinov, M., \& Sporns, O. (2010). Complex network measures of brain connectivity: Uses and interpretations. Neuroimage, 52, 1059-1069. 
40. Benjamini, Y., \& Hochberg, Y. (1995). Controlling the false discovery rate: A practical and powerful approach to multiple testing. Journal of the Royal Statistical Society Series B, 57, 289-300.

41. Wechsler, D. (1999). Wechsler Abbreviated Scale of Intelligence (WASI; Wechsler, 1999). Minneapolis, MN: Pearson Assessments.

42. Gratz, K. L., \& Roemer, L. (2004). Multidimensional assessment of emotion regulation and dysregulation: Development, factor structure, and initial validation of the difficulties in emotion regulation scale. Journal of Psychopathology and Behavioral Assessment, 26, 41-54.

43. Williams, L. M., Sidis, A., Gordon, E., \& Meares, R. A. (2006). "Missing links" in borderline personality disorder: loss of neural synchrony relates to lack of emotion regulation and impulse control. Journal of Psychiatry and Neuroscience, 31, 181-188.

44. Fries, P. (2005). A mechanism for cognitive dynamics: neuronal communication through neuronal coherence. Trends in Cognitive Sciences, 9, 474-480.

45. Womelsdorf, T., Schoffelen, J.M., Oostenveld, R., Singer, W., Desimone, R., Engel, A.K., \& Fries, P. (2007). Modulation of neuronal interactions through neuronal synchronization. Science, 316, 1609-1612.

46. Adolphs, R. (2002). Neural systems for recognizing emotion. Current Opinion in Neurobiology, 12, 169-177.

47. Bush, G., Luu, P., \& Posner, M. I. (2000). Cognitive and emotional influences in anterior cingulate cortex. Trends in Cognitive Sciences, 4, 215-222.

48. Ochsner, K.N., Gross, J.J., 2004. Thinking makes it so: a social cognitive neuroscience approach to emotion regulation. In: Vohs, K., Baumeister, R., (Eds.), The Handbook of SelfRegulation. Erlbaum, NJ. 
49. Winsper, C., Lereya, S. T., Marwaha, S., Thompson, A., Eyden, J., \& Singh, S. P. (2016). The aetiological and psychopathological validity of borderline personality disorder in youth: A systematic review and meta-analysis. Clinical Psychology Review, 44, 13-24.

50. Goodman, M., Hazlett, E. A., Avedon, J. B., Siever, D. R., Chu, K. W., \& New, A. S. (2011). Anterior cingulate volume reduction in adolescents with borderline personality disorder and comorbid major depression. Journal of Psychiatric Research, 45, 803-807.

51. Segall, J. M., Allen, E. A., Jung, R. E., Erhardt, E. B., Arja, S. K., Kiehl, K. A., \& Calhoun, V. D. (2012). Correspondence between structure and function in the human brain at rest. Frontiers in Neuroinformatics, 6, 10.

52. Palva, S., \& Palva, J. M. (2007). New vistas for $\alpha$-frequency band oscillations. Trends in Neurosciences, 30, 150-158.

53. Palva, S., \& Palva, J. M. (2011). Functional roles of alpha-band phase synchronization in local and large-scale cortical networks. Frontiers in Psychology, 2, 204.

54. Bar, M., Kassam, K. S., Ghuman, A. S., Boshyan, J., Schmid, A. M., Dale, A. M., Hamalainen, M. S., Marinkovic, K., Schacter, D. L., Rosen, B. R. \& Halgren, E. (2006). Top-down facilitation of visual recognition. Proc. Natl. Acad. Sci. U.S.A. 103, 449-454.

55. Freunberger, R., Klimesch, W., Griesmayr, B., Sauseng, P., and Gruber, W. (2008). Alpha phase coupling reflects object recognition. Neuroimage 42, 928-935.

56. Mima, T., Oluwatimilehin, T., Hiraoka, T., \& Hallett, M. (2001). Transient interhemispheric neuronal synchrony correlates with object recognition. Journal of Neuroscience, 21, 3942-3948. 